\title{
Direct Backward-Emitted Third-Harmonic Generation and Its Application to Clinical Microscopy
}

\author{
Szu-Yu Chen, Shih-Peng Tai, Tsung-Han Tsai, and Chi-Kuang Sun \\ Graduate Institute of Electro-Optical Engineering and Department of Electrical Engineering, National Taiwan University, Taipei, 10617 TAIWAN, \\ R.O.C. \\ Tel:+886-2-23659703. FAX:+886-2-23677467. Email: f92941004@ntu.edu.tw.
}

\begin{abstract}
Similar to forward-emitted third-harmonic-generation (THG), backward-emitted THG is found to be sensitive to interface inhomogeneity with improved longitudinal sectioning capability and can be applied to noninvasive clinical imaging.

C2005 Optical Society of America

OCIS codes: (170.3880) Medical and biological imaging, (190.4160) Multiharmonic generation
\end{abstract}

Third harmonic generation (THG) microscopy, with a high sensitivity to interfaces [1], has been applied to a variety of biology studies [2], including developmental biological study of zebrafish embryos and $\mathrm{Ca}^{2+}$ wave imaging. THG is known to leave no energy deposition on the interacted materials due to its virtual-level transition characteristic and can thus provide a noninvasive way for biological imaging without photodamage and photobleaching. By utilizing the intrinsic nonlinearity provided by the biological samples, high optical sectioning power with a submicron 3D spatial resolution can be achieved. Due to the momentum conservation law, all previous THG microscopes were constructed with a forward collection geometry. With a limited penetration capability, this transmission-type geometry of the demonstrated THG microscopy restricts its application to many biological and clinical studies, in which the samples are too thick for the THG signals to go through. Especially in clinical diagnoses, backward collection geometry is required due to the thickness of human body. In this talk, we demonstrate theoretically and experimentally that significant backward emitted THG occurs at a thin film or at interfaces of two materials with discontinuous susceptibility. Due to destructive interference effects, the backward emitted THG is found to have an improved longitudinal sectioning capability compared with the forward emitted THG. A THG microscope was then constructed with a backward collection geometry while 3D THG images of cell membranes and structural interfaces deep inside biological samples will also be presented in the conference.

First consider a strongly focused Gaussian beam propagation in the $+z$ direction at an angular frequency $\omega$ with an electric field intensity distribution of

$$
E_{\omega}(x, y, z)=\frac{A_{\omega}}{1+i \frac{z}{b}} e^{\frac{x^{2}+y^{2}}{w_{\omega \infty}^{2}\left(1+i \frac{z}{b}\right)}},
$$

where $\mathrm{b}$ is the confocal parameter, $w_{\omega o}$ is the beam waist of the Gaussian beam, and $A_{\omega}$ is a constant. Through third harmonic generation susceptibility $\chi^{(3)}(3 \omega: \omega, \omega, \omega)$, similar to previous derivation of the forward THG [3], the steady state paraxial wave equation for the electric field intensity of the backward emitted third harmonic wave $E_{3 \omega}$ at an angular frequency of $3 \omega$ can be derived as:

$$
\left[\frac{-1}{i 2 k} \nabla_{T}^{2}+\frac{\partial}{\partial z}\right] E_{3 \omega}(x, y, z)=-\frac{i 3 \omega}{8 c n(3 \omega)} \chi^{(3)}(z) E_{\omega}^{3}(x, y, z) e^{i \Delta k z}
$$

where $\Delta k=3 k(\omega)+k(3 \omega)$ is the phase difference between fundamental and the backward emitted THG, c is the speed of light in vacuum, and $n(3 \omega)$ is the refractive index of the backward THG in the media. The backward emitted intensity of $E_{3 \omega}(-\infty)$ can be found to be proportional to $|J(-\infty)|^{2}$, where

$$
\mathrm{J}(-\infty)=\int_{\infty}^{-\infty} d z^{\prime} \frac{\chi^{(3)}\left(\mathrm{z}^{\prime}\right) e^{i \Delta k z^{\prime}}}{\left(1+i \frac{z^{\prime}}{b}\right)^{2}}
$$




\section{QML3}

Now consider two different cases with (A) an interface between a material and vacuum and (B) a $50 \mathrm{~nm}$-thick thin film in vacuum with different susceptibility. The numerical calculated backward THG (B-THG) intensities as a function of Gaussian beam foci position $\mathrm{z}$ shown in Figure 1. The media are both assumed to be isotropic with a refractive index 1.5 for both fundamental and third harmonic generation wavelengths, and the confocal parameter is set to be $1.36 \mu \mathrm{m}$ to simulate the effect of an NA 0.9 objective at a fundamental wavelength of $1230 \mathrm{~nm}$. It is noted that the B-THG signals in both cased shown peaks at the interfaces, where the focusing position is set to be zero. Our results thus indicate that B-THG is highly sensitive to interface where the momentum conservation law is broken. Thus, by collecting B-THG signals with a strongly focused Gaussian beam, information of the interfaces and structures of the samples can be revealed. Moreover, by comparing the simulated B-THG results with that of forward THG (F-THG), it can be found the longitudinal sectioning capability of B-THG is better than that of the FTHG (with a narrower spatial width in Fig. 1), due to the strong destructive interference effect.
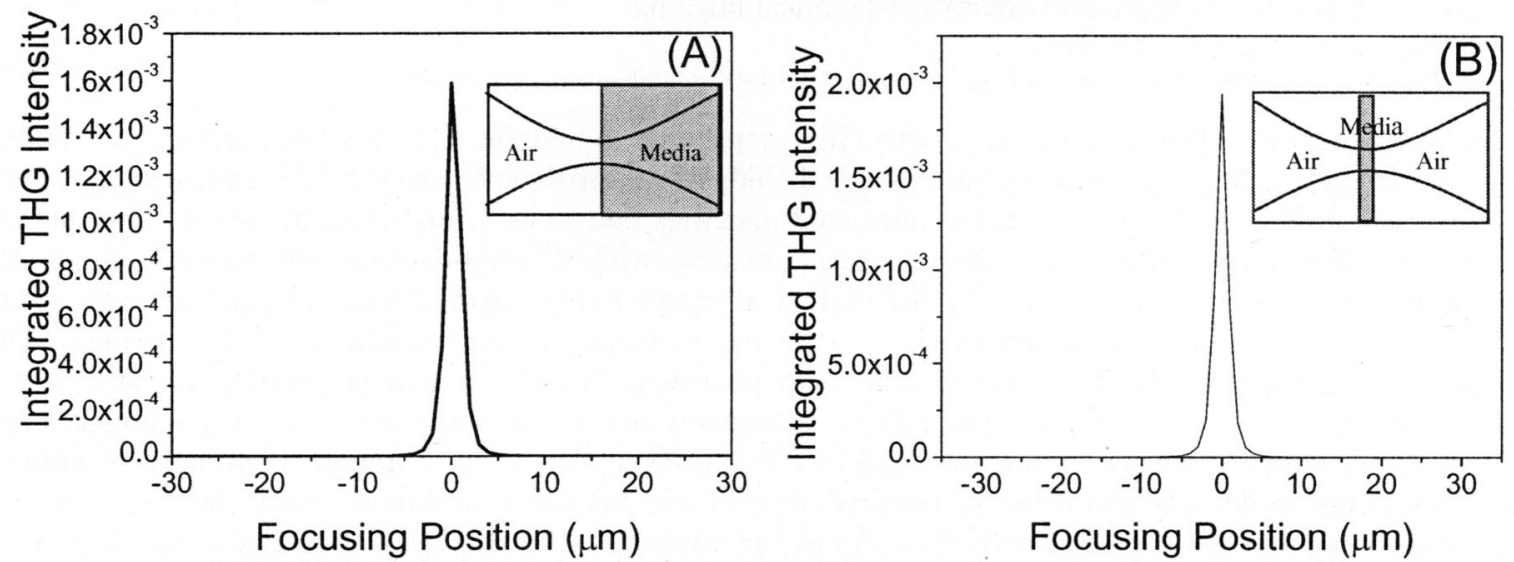

Figure 1 The numerical calculated B-THG intensities as a function of Gaussian beam foci position $\mathrm{z}$ for (A) an interface between a material and vacuum and (B) a 50nm-thick thin film in vacuum. The media are both assumed to be isotropic with $n(\omega)=n(3 \omega)=1.5$ and the confocal parameter of the laser beam is set to be $1.36 \mu \mathrm{m}$ with fundamental wavelength of $1.23-\mu \mathrm{m}$. The interfaces are located at position zero.

To experimentally confirm our calculation, we have conducted a B-THG experiment on the glass surface. A femtosecond $\mathrm{Cr}$ :forsterite laser was directed into a scanning microscope and focused by an objective with different numerical apertures on the glass of $1.5 \mathrm{~mm}$ thickness. The geometry was arranged so that the reflection from the underlying surface (about $1.5 \mathrm{~mm}$ away) of the generated F-THG could be neglected due to a confocal geometry. By analyzing the intensity of B-THG and F-THG signals at different depth and by changing the confocal parameters, the experiment results match the theory qualitatively, supporting the direct backward mission mechanism and its improved longitudinal resolution.
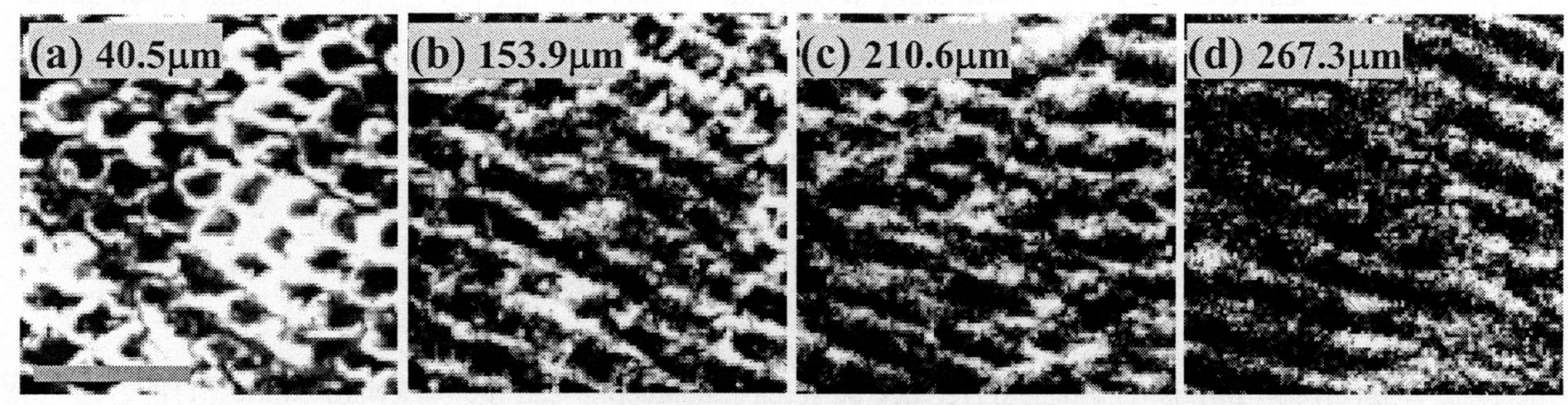

Figure 2 B-THG images obtained by directly scanning downward from the surface of a human tooth at focusing depths of (a) $40.5 \mu \mathrm{m}$; (b) $153.9 \mu \mathrm{m}$; (c) $210.6 \mu \mathrm{m}$; (d) $267.3 \mu \mathrm{m}$ from the surface. Scale bar: $20 \mu \mathrm{m}$

A THG microscope was then constructed with a backward collection geometry to demonstrate 3D biological imaging. Fig. 2 is an example series of B-THG images obtained from different depths of the enamel part of a human tooth, which is too thick for THG to penetrate. The enamel, which is the surface layer responsible for preventing the live parts of tooth from attacking by the bacteria or acids, is made up of inorganic micro-prisms and the directions of the micro-prisms continuously change with the depth from the surface. Since the enamel part is the first outer layer of a tooth, by scanning the laser beam under the tooth surface and collecting the B-THG signals we can resolve the 


\section{QML3}

interfaces between micro-prims. The structure variation with depth inside the enamel can thus be noninvasively observed by changing the focusing depth of the scanning laser beam. Fig. 2(a) was obtained at a depth of $40.5-\mu \mathrm{m}$ from the surface, while the sketch of each prism can be clearly revealed. The 3D prism arrangement can be optically resolved even with a depth of over $300-\mu \mathrm{m}$ from the surface.

In summary, we have demonstrated theoretically and experimentally that significant backward emitted THG occurs at a thin film or at interfaces of two materials with discontinuous susceptibility. Due to destructive interference effects, the backward emitted THG was found to provide an improved longitudinal sectioning capability compared with the forward THG, which is already better than the traditional two-photon fluorescence. A THG microscope was constructed with a backward collection geometry while 3D THG images of cell membranes and structural interfaces deep inside biological samples will be presented in the conference. This new founding shows great promises for future noninvasive clinical optical imaging. This work is sponsored by National Health Research Institute of Taiwan with NHRI-EX93-9201EI and NTU Center for Genome Medicine.

\section{Reference}

[1] Barad Y et al. "Nonlinear scanning laser microscopy by third harmonic generation." Appl. Phys. Lett. 70, 922-924 (1997)

[2] Yelin D et al. "Laser scanning third-harmonic-generation microscopy in biology." Opt. Express 5, 169-175 (1999)

[3] J.M. Schins et al. "Determination of material properties by use of third-harmonic generation microscopy." J. Opt. Soc. Am. B 19, 1627-1634 (2002) 